\title{
INFLUENCIA DEL CAUTIVERIO EN EL COMPORTAMIENTO Y EN LOS NIVELES DE CORTISOL DEL MONO TITI GRIS (Saguinus leucopus)
}

\author{
INFLUENCE OF CAPTIVITY IN BEHAVIOR AND CORTISOL LEVELS OF TITI GREY \\ MONKEY (Saguinus leucopus)
}

\begin{abstract}
RESUMEN
La especie Saguinus leucopus está catalogada como especie en peligro de extinción debido a su escasa distribución y a la destrucción de su hábitat. Su situación de peligro sirvió de justificación para determinar la influencia del cautiverio en los patrones comportamentales y en los niveles de cortisol. Es pertinente el análisis de cortisol y el comportamental, ya que permiten establecer las conductas propias de la especie en situación de estrés y posibilitan la implementación de estrategias favoreciendo el bienestar de los individuos, su reproducción en cautiverio y reintroducción a la vida natural. Metodológicamente se seleccionaron dos encierros el zoológico de Santacruz (con siete individuos en un núcleo familiar) y el zoológico de Piscilago (con dos individuos reunidos con anterioridad); se realizaron etogramas durante 19 días, contabilizando las frecuencias y secuencias comportamentales. Simultáneamente se estimaron las concentraciones de cortisol fecal por medio del Ensayo por Inmunoabsorción Ligado a Enzimas (ELISA), con el fin de hallar alguna posible asociación con el comportamiento en cuanto a la presencia de conductas repetitivas y el aumento del cortisol como posible indicador de estrés. Nuestros resultados muestran que las conductas estereotipadas fueron exploración y / o reconocimiento y se hallaron los estresores en el comportamiento de locomoción. Finalmente, los valores de cortisol fueron mayores en Piscilago (215,5 ng / g) que en Santacruz (145 ng / g), concluyéndose que las estereotipias están asociadas a los niveles de cortisol, como se muestra en la unidad comportamental Lleva (patrón de locomoción), que mostró una dependencia frente a la hormona y unos altos niveles de ésta.
\end{abstract}

PALABRAS CLAVE: conductas estereotipadas; etograma; hormona del estrés; secuencias comportamentales; primates; cautiverio

\begin{abstract}
White-footed tamarin is listed as endangered species because of its limited distribution and the destruction of their habitat. Its danger served as an excuse to determine the influence of captivity in the behavioral patterns and levels of cortisol. It is pertinent analysis of cortisol and behavioral as possible to establish their own behavior of the species under stress and allow the implementation of strategies favoring the well-being of individuals, captive breeding and reintroduction to natural life. Methodologically two runnings Zoo Santacruz (seven individuals in a household) and Zoo Piscilago (with two individuals together previously) were selected; ethograms were held for 19 days, counting the frequency and behavioral sequences. Simultaneously fecal cortisol concentrations were estimated linked immunosorbent assay Enzyme (ELISA), in order to find a possible association with behavior in the presence of repetitive behaviors and increased cortisol as a possible indicator of stress. Our results show that stereotypical behaviors were exploration and / or recognition, and stressors were found in the behavior of locomotion. Finally, cortisol levels were higher in Piscilago (215.5 ng / g, Santacruz $145 \mathrm{ng} / \mathrm{g}$ ), concluding that the stereotypes are associated with cortisol levels, as shown in behavioral unit carries (pattern of locomotion) that showed a hormone dependence and high levels of this.
\end{abstract}

KEY WORDS: stereotypical behaviors; ethogram; stress hormone; behavioral sequences; primates; captivity 
Debido a problemáticas como el trafico ilegal y la rápida fragmentación de su habitat, Saguinus leucopus es considerada una especie en peligro (Morales-Jiménez et al., 2008), muchos individuos de esta especie se encuentren en condiciones de cautiverio, lo que pueden incrementar los niveles de glucocorticoides liberados. Esto repercute en la salud y bienestar de los individuos, al ocasionar cambios en el comportamiento normal de la especie y aumentar los niveles normales de estrés en ellos, como lo establece De Camargo (2004).

Por esta razón se hizo necesario plantear un método de diagnóstico del estrés, que determinara cómo el tipo de cautiverio influye en el comportamiento y supervivencia de los individuos, a partir de métodos no invasivos como catálogos de comportamiento y la medición de los niveles de cortisol en excretas de Saguinus leucopus. Estos métodos se consolidan como herramienta útil al no alterar físicamente a los individuos y permitir la indagación de concentraciones hormonales (Valdespino et al., 2007) en excretas. El muestreo de heces fecales se combinó con la elaboración de un catálogo comportamental que permitiera analizar conductas estereotipadas, posibles estresores y su relación con los niveles de cortisol en el mono titi gris.

Para establecer el aumento del estrés y la posible relación entre el comportamiento atípico y la liberación del glucocorticoide se contrastó el análisis de dos sitios de muestreo presentes en zoológicos. El Zoológico de Santacruz (sin acceso de turistas) y en el Zoológico de Piscilago (con acceso de turistas), ambos ubicados en Cundinamarca.

Para esto, primero se observaron las condiciones físicas del encierro, se realizó una descripción y un esquema de cada sitio de muestreo, se hizo énfasis en el tipo de enriquecimiento de cada uno, las dimensiones de la jaula y el número de individuos por jaula. El encierro del zoológico de Santacruz con un tamaño de $9 \mathrm{~m}^{2}$ en el que se encontraban siete individuos (cinco adultos: tres machos, dos hembras y dos crías) configurados como núcleo familiar y el otro en el zoológico de Piscilago de $10,4 \mathrm{~m}^{2}$ con 2 individuos reunidos con anterioridad.

Luego se desarrolló el muestreo comportamental durante 19 días en ambos encierros que conllevó a la realización de un etograma mediante muestreo ad libitum con registro continuo y animal focal. Los periodos de observación fueron de 15 minutos, seguidos de cinco minutos de descanso, como establece Leal (2004), se realizó conteo de frecuencias, la observación de las secuencias comportamentales y sus respectivos análisis estadísticos mediante chi-cuadrado, alternándose con el muestro fecal. Las heces colectadas (8 para cada encierro) fueron analizadas mediante ELISA (en la Fundación Colombiana de Estudios de Parásitos FUNCEP), para hallar en ellas los niveles de cortisol y buscar la posible asociación con el comportamiento a través de la prueba en mención.

Durante la elaboración del catálogo comportamental se utilizó una prueba de independencia de las unidades comportamentales y las frecuencias de dichas unidades para cada uno de los encierros, y se midió el grado de asociación entre los comportamientos y las frecuencias obtenidas para cada muestreo. En el análisis de frecuencias comportamentales o el establecimiento de las diadas (secuencias) se realizó una la prueba chi-cuadrado con el fin de hallar las probabilidades de dicha dependencia para cada zoológico, además de establecer una comparación de las diadas en cada encierro y reconocer los posibles estresores a partir de la independencia o no de estas (Tabla 1).

Las unidades comportamentales comprendidas dentro del patrón de Exploración y / o Reconocimiento fueron las más abundantes en cuanto a las unidades estereotipadas o exageradas ("Las estereotipias o comportamientos estereotipados son definidos tradicionalmente como repetitivos, invariables y sin función u objetivo aparente" Rushen y Mason, 2006). Además de obtenerse las unidades adscritas a la Locomoción como las de mayor ocurrencia o abundancia.

Estas conductas estereotipadas están asociadas a ambientes no óptimos y son utilizadas como indicador de bienestar según Manson (1991) y desembocan en estrés en cautiverio, comportamientos estereotipados, como lo explica Erwin y Deni (1979) y en una disrupción de la homeostasis fisiológica, evidenciada en las variaciones de los niveles de cortisol. En la misma línea los posibles estresores que se obtuvieron a partir del análisis de las diadas comportamentales fueron Salto-Lleva, Desplazarse por soga-Frotarse la cara en el encierro del Zoológico de Santacruz, y Salto-Tomar alimento con la boca, SaltoDesplazarse por el suelo y Desplazarse por soga-Frotarse la cara en el Zoológico de Piscilago principalmente. 
Tabla 1. Posibles estresores dentro del catálogo comportamental. Nivel de asociación p $\leq 0,05$.

\begin{tabular}{llcc}
\hline \multirow{2}{*}{ Comportamiento } & \multicolumn{1}{c}{ Diada } & Z. Santacruz & Z. Piscilago \\
\cline { 3 - 4 } & & chi-cuadrado & chi-cuadrado \\
\hline & salto- lleva & 0,01 & ---- \\
& Salto-tomar alim. con boca & 0,18 & 0,0060 \\
& salto-desp.suelo & ---- & 0,020 \\
Locomoción & desp. por soga-frotarse la cara & 0,05 & 0 \\
& desp. por reja-tomar alim.boca & 0,01 & 88,17 \\
& comer 1 mano-desp.por reja & 0,0064 & ----- \\
Alimentación-Social & frotarse cuerpo-salto & ---- & 0,034 \\
& frotarse cuerpo-aci.individual & $-\cdots,-$ & 0,001 \\
& ob desde reja-frotarse la cara & ---- & 0,05 \\
Exploración y & ob desde rama-desp. por reja & 0,0002 & 5,32 \\
Reconocimiento & columpiarse-aci.social & 0,03 & ----- \\
\hline
\end{tabular}

Las ocho muestras de materias fecales recolectadas del animal focal en cada zoológico, se introdujeron en tubos con alcohol etílico al $95 \%$, para luego ser analizados mediante pruebas inmuno-absorbentes de unión enzimática (ELISA). Los resultados mostraron que las concentraciones de cortisol (ng / g) fueron mayores en la mayoría de los casos para el encierro de Piscilago y menores para Santacruz.

Mientras el promedio de cortisol en Santacruz fue de 145 ng / g, con máximos de hasta 526 ng / g, mínimos de 20 ng / g y una desviación estándar de 172,6, en Piscilago el promedio fue de $215,5 \mathrm{ng} / \mathrm{g}$ con incrementos hasta de 648 ng / g, mínimos de 76 ng / g y con desviación estándar de 177,5 (Figura 1), a partir de este análisis se realizó la comparación de las concentraciones de cortisol en el encierro de Santacruz y de Piscilago con niveles de cortisol en especies cercanas como Saguinus oedipus (Linnaeus, 1758), cuyo valor de la hormona se encuentra en un rango de 570 ng / g según Sodaro y Saunders (1999) (Tabla 2).

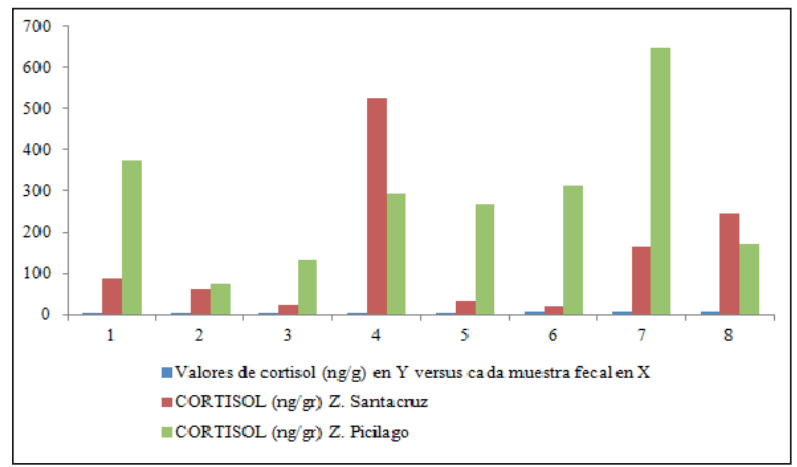

Figura 1. Niveles máximos y mínimos del cortisol (ng / g) para individuos de Saguinus leucopus en el encierro de Santacruz y de Piscilago. La desviación estándar para los datos recogidos en Piscilago es 177,5 con una media de 284,5 mientras que los datos de Santacruz se observa una desviación estándar de 172,6 con una media de 145.

Según la prueba de chi-cuadrado, la frecuencia mínima esperada fue de 0,13 , y los valores de la mayoría de 
comportamientos frente al cortisol son superiores a este dato, indicando que estos son independientes de los niveles de cortisol del individuo. Sin embargo para la unidad comportamental denominada Lleva la cual se describe como perseguir y escapar de otros mediante el desplazamiento veloz por soga, reja, rama y saltos y presentada únicamente en el encierro ubicado en el zoológico de Santacruz se encontró que sí se presenta una dependencia frente a los niveles de cortisol al arrojarse un resultado de 0,039, menor que la frecuencia mínima esperada de 0,13 .

Tabla 2. Concentraciones de cortisol (hormona del estrés) en el encierro de Santacruz y de Piscilago y comparación con niveles de cortisol en especies cercanas y primates del viejo mundo como referencia de valores esperados en el género.

\begin{tabular}{ccccc}
\hline & \multicolumn{2}{c}{ Saguinus leucopus } & $\begin{array}{c}\text { Saguinus oedipus } \\
\text { (Linnaeus, 1758) }\end{array}$ & $\begin{array}{c}\text { Primates del viejo } \\
\text { mundo }\end{array}$ \\
\hline No. de muestra fecal & $\begin{array}{c}\text { Cortisol (ng / g) Z. } \\
\text { Santacruz }\end{array}$ & $\begin{array}{c}\text { Cortisol (ng / g) Z. } \\
\text { Piscilago }\end{array}$ & Cortisol (ng / g) & Cortisol (ng / g) \\
\hline 1 & 88 & 374 & & \\
2 & 62 & 76 & & \\
3 & 22 & 132 & 570 & 108,738 \\
4 & 526 & 294 & & \\
5 & 34 & 268 & & $1986)$ \\
6 & 20 & 312 & & \\
7 & 164 & 648 & & \\
8 & 244 & 172 & & \\
\end{tabular}

En conclusión el cortisol puede tener cierto grado de asociación con los comportamientos y esto conduce a pensar, que al no tener las condiciones adecuadas en el encierro los individuos llegan a verse afectados tanto a nivel comportamental como fisiológico como sucedió en el zoológico de Piscilago, donde los monos aunque no eran de exhibición se encontraban ubicados en un sitio de confluencia constante de turistas, a diferencia del encierro de Santacruz, siendo posible afirmar que la presencia de los turistas tiene un efecto significativo en el aumento del cortisol, y que ni el sexo, ni el máximo mensual de la temperatura, influye significativamente en los niveles de esta hormona (Behie et al., 2010).

Así, estímulos conductuales como Lleva al presentar una duración prolongada y real asociación con los niveles de cortisol puede ser catalogado como un estímulo que por su duración se consideraría negativo al no ser breve y repetirse continuamente, como establece Brousset et al. (2005). Este factor conductual perturbaría la homeostasis de los individuos consolidándose como respuesta al estrés y reacción de defensa de los monos al aumento del glucocorticoide en mención (Möstl y Palme, 2002).
Así, el incremento de cortisol en los individuos por un tiempo prolongado puede incurrir en efectos negativos en la salud del organismo al suprimir el sistema inmune y aumentar el metabolismo de las proteínas, lo cual afecta el crecimiento del animal como propone Martínez-Mota et al. (2007). Por último como se mencionó anteriormente, el encierro de Picilago donde se presentó el mayo valor de cortisol, si bien no era parte de la exhibición del zoológico, si estaba ubicado en un sitio con acceso constante de turistas lo cual probablemente desencadenaría el aumento de la hormona, que como establece Rangel-Negrín et al. (2009) la presencia de estrés y el aumento del cortisol se evidencia en monos que habitan en áreas protegidas o no, pero en las cuales hay perturbación continua del hombre, esto como explica el mismo autor conduciría a la falta de viabilidad a largo plazo, la disminución de la población y la extinción de la especie.

Los bajos niveles de cortisol en el encierro de Santacruz a diferencia de los de Piscilago pueden deberse a que el grupo de siete individuos se constituye en núcleo familiar y no han sufrido separación y a que su encierro no presenta invasión del hombre, a diferencia de los dos 
individuos de Piscilago, que en meses anteriores del estudio, habían sufrido separación social y reubicación en un nuevo encierro por causas reproductivas.

Finalmente, se recomienda el uso de los datos comportamentales obtenidos aquí para Saguinus leucopus como base en la comparación con los arrojados en otros cautiverios y en vida libre, al igual que los de cortisol, ya que no hay registros anteriores de los niveles de esta hormona tomados mediante muestreo no invasivos ni en cautiverio, ni en vida libre.

\section{BIBLIOGRAFÍA}

Behie, A.M., Pavelka, M.S.M. y Chapman, C.A. 2010. Sources of variation in fecal cortisol levels in howler monkeys in Belize. American Journal of Primatology 72(7): 600-606.

Brousset, M., Galindo, F., Valdez, R., Romano, M. y Schuneman, A. 2005. Cortisol en saliva, orina y heces: evaluación no invasiva en mamíferos silvestres. Veterinaria México 36(3): 325-337.

De Camargo, B.S. 2004. Estrés, síndrome general de adaptación o reacción general de alarma. Revista Médico Científica 17(2): 78-86.

Erwin, J. y Deni, R. 1979. Strangers in a strange land: abnormal behaviors and abnormal environments. En: Erwin, J., Maple, T.L. y Mitchell, G., Editores. Captivity and behavior: Primates in breeding colonies, laboratories and zoos. Van Nostrand Reinhold, New York.

Klosterman, L.L., Murai, J.T. y Siiteri, P.K. 1986. Cortisol levels, binding, and properties of corticosteroid-binding globulin in the serum of primates. Endocrinology 118(1): 424-434.

Leal, A.M. 2004. Identificación de los patrones de comportamiento y conformación de un grupo social de tití gris (Saguinus leucopus) en proceso de rehabilitación.
Tesis de pregrado. Universidad Distrital Francisco José de Caldas, Bogotá, Colombia.

Manson, G. 1991. Stereotypes: a critical review. Animal behavior 41: 1015-1037.

Martínez-Mota, R., Valdespino, C., Sanchez-Ramos, M.A. y Serio-Silva, J.C. 2007. Effects of forest fragmentation on the physiological stress response of black howler monkeys. Animal Conservation 10: 374-379.

Morales-Jiménez, A.L., Vejarano, S., Rodríguez, C.L. y Ospina, O. 2008. Programa Nacional para la Conservación de la Especie Endémica de Colombia tití gris (Saguinus leucopus). CORPOCALDAS Ministerio de Ambiente Vivienda y Desarrollo Territorial Fundación Biodiversa Colombia Asociación Colombina de Primatología, Bogotá, Colombia.

Möstl. E. y Palme, R. 2002. Hormones as indicators of stress. Domestic Animal Endocrinology 23: 67-74.

Rangel-Negrín, A., Alfaro, J.L., Valdez, R.A., Romano, M.C. y Serio-Silva, J.C. 2009. Stress in Yucatan spider monkeys: effects of environmental conditions on fecal cortisol levels in wild and captive populations. Animal Conservation 12(5): 496-502.

Rushen, J. y Mason, G. 2006. A decade-or-more's progress in understanding stereotypic behaviour. En: Mason, G. y Rushen, J., Editores. Stereotypic Animal Behaviour: Fundamentals and Applications to Welfare. Second edition. CABI, Wallingford, UK.

Sodaro, V. y Saunders, N. 1999. Callitrichid Husbandry Manual. Neotropical Primate Taxon Advisory Group, Chicago, United States.

Valdespino, C., Martínez, R., García, L.M. y Martínez, L.E. 2007. Evaluación de los eventos reproductivos y estrés fisiológico en vertebrados silvestres a partir de sus excretas: evolución de una metodología no invasiva. Acta Zoológica Mexicana 23(3): 151-180.

Fecha de recepción: 11/11/2015

Fecha de aceptación: 03/11/2016

Publicado en línea: 07/04/2017

Para citar este artículo: Estefenn-Barbosa, V. y Gómez, Y.Y. 2017. Influencia del cautiverio en el comportamiento y en los niveles de cortisol del mono titi gris (Saguinus leucopus). Revista Intropica Vol. 12(1): 55 - 59. DOI: http://dx.doi.org/10.21676/23897864.2036 